\title{
Time Reduction of New Patient Consultation at the Department of Ophthalmology and Visual Sciences of a Philippine Tertiary Hospital
}

\author{
George Michael N. Sosuan, MD, Antonio Niccolo D.L. Agustin, MD, Roland Joseph D. Tan, MD, MS, \\ Rolando Enrique D. Domingo, MD and Marissa N. Valbuena, MD, MHPEd \\ Department of Ophthalmology and Visual Sciences, College of Medicine and Philippine General Hospital, University of the Philippines Manila
}

\begin{abstract}
Objective. To decrease the total time spent of new patients on a General Clinic consult at the Department of Ophthalmology and Visual Sciences of a Philippine Tertiary Hospital.

Methods. A time quality management team was formed. The description of the process of a General Clinic new patient consult was elucidated and was consolidated in a data collection form. Convenience sampling of the population was done. The collection and analysis of the data were done with institution of interventions to address the factors causing the prolonged consultation visit; then, pre-intervention analysis, post-intervention analysis and comparison were done.

Results. Thirty-five new patients were tracked prior to and after intervention. Among the identified causes for prolonged new patient consult were delay in temporary chart, front of chart and blue card issuance, insufficient examination tools and resident dedicated to the General Clinic, unnecessary examination and patient not being around when called. Most causes were addressed. A mean decrease of $68 \pm 112$ minutes or approximately $18 \%$ in total time stay was noted.

Conclusion. This study showed that the total consultation time of a new patient in General Clinic decreased. This was achieved with the help of most of the personnel involved in the system after identifying factors causing the prolonged consultation visit and instituting interventions to address these identified factors. The improvement in health service delivery was taken as a step by step process. A preliminary step was demonstrated in this paper for future interventions for better service delivery.
\end{abstract}

Keywords: Time and Motion Study, New Patient, Ophthalmology, Out-Patient, Consultation

\section{INTRODUCTION}

The Out-Patient General Clinic of the Department of Ophthalmology and Visual Sciences of a Philippine Tertiary Hospital had an average of 15,000 new consults annually prior to the COVID-19 pandemic. An increase in the number of new patient consults was noted each year, but the facilities, waiting area, and manpower remained the same. This has resulted to crowded areas which often lead prolonged waiting time. ${ }^{1}$ Informal complaints regarding service delivery from patients arose, often with the prolonged waiting time. The General Clinic thus required a systematic study of its services for its efficient management and function. It was found imperative that a time and motion study of the General Clinic be conducted. Appropriate inexpensive interventions could go a long way in improving efficiency of the system..$^{2-5}$

The time and motion study is a scientific study of finding the most efficient method of doing a task. It is a helpful way

Corresponding author: Roland Joseph D. Tan, MD, MS Department of Ophthalmology and Visual Sciences College of Medicine and Philippine General Hospital University of the Philippines Manila

Taft Avenue, Ermita, Manila 1000, Philippines

Email:rdtan@up.edu.ph 
to find a best method of doing a work, and to assist effectively in managing and controlling its activity. ${ }^{1,2}$ In time and motion studies, an external observer captures detailed data on the movements and duration required to accomplish a task followed by analysis focused on improving efficiency. ${ }^{3}$ The principal philosophy of this kind of study is that (a) although there are numerous ways to perform any task, one method will be superior to others, and (b) the superior method can be determined by observing and analyzing the time taken to carry out parts of the activity. ${ }^{4}$

In the health care settings, service delivery must be given in the most efficient way without compromising quality of care to have a satisfied patient. This study aimed to decrease the total time spent of new patients on a General Clinic consult at the department. Informal complaints on service delivery from patients had been arising, particularly with the prolonged waiting time. By identifying the factors causing the prolonged new patient consult, the department can institute changes to decrease the total time spent in a new General Clinic consultation visit and maximizing patient satisfaction in the health service delivery.

\section{METHODOLOGY}

A time quality management team was formed composed of the Department Chair, a time quality management specialist, the Chief Resident, a resident, a General Clinic nurse and a General Clinic nursing aid. The steps every new patient of the General Clinic undertakes were identified and were listed by the team. The team identified 7 steps that a new patient undergoes in a usual General Clinic consult (Figure 1).

A data collection form was used to track each patient and to document the time in minutes each step would take. The additional details per step were also noted in the data collection form. Convenience sampling was done for 10 days which included two Mondays, two Tuesdays, two Wednesdays, two Thursdays, and two Fridays. There was only one instructed and trained external data collector for the study. The General Clinic personnel were not aware that the patient encounters were being observed. At the end of data collection, the data was analyzed and was presented to the team. Root cause analysis of the causes of prolonged consultation visit was done. The team discussed and identified barriers to efficiency. Interventions were contemplated and implemented. Four months after initiation of intervention, a post-intervention convenience sampling data collection for 10 days was done in the same way as the pre-intervention sampling. Data analysis and comparison of the preintervention and post-intervention groups were done. Time reduction was defined as the median of the values computed using the formula below:
Data were tabulated and subjected to Mann-Whitney U Test using Microsoft Excel version 2016 (Microsoft Corporation, Redmond, Washington, USA) and Stata 14 (Statacorp, College Station, Texas, USA). A p-value of 0.5 or less is considered statistically significant.

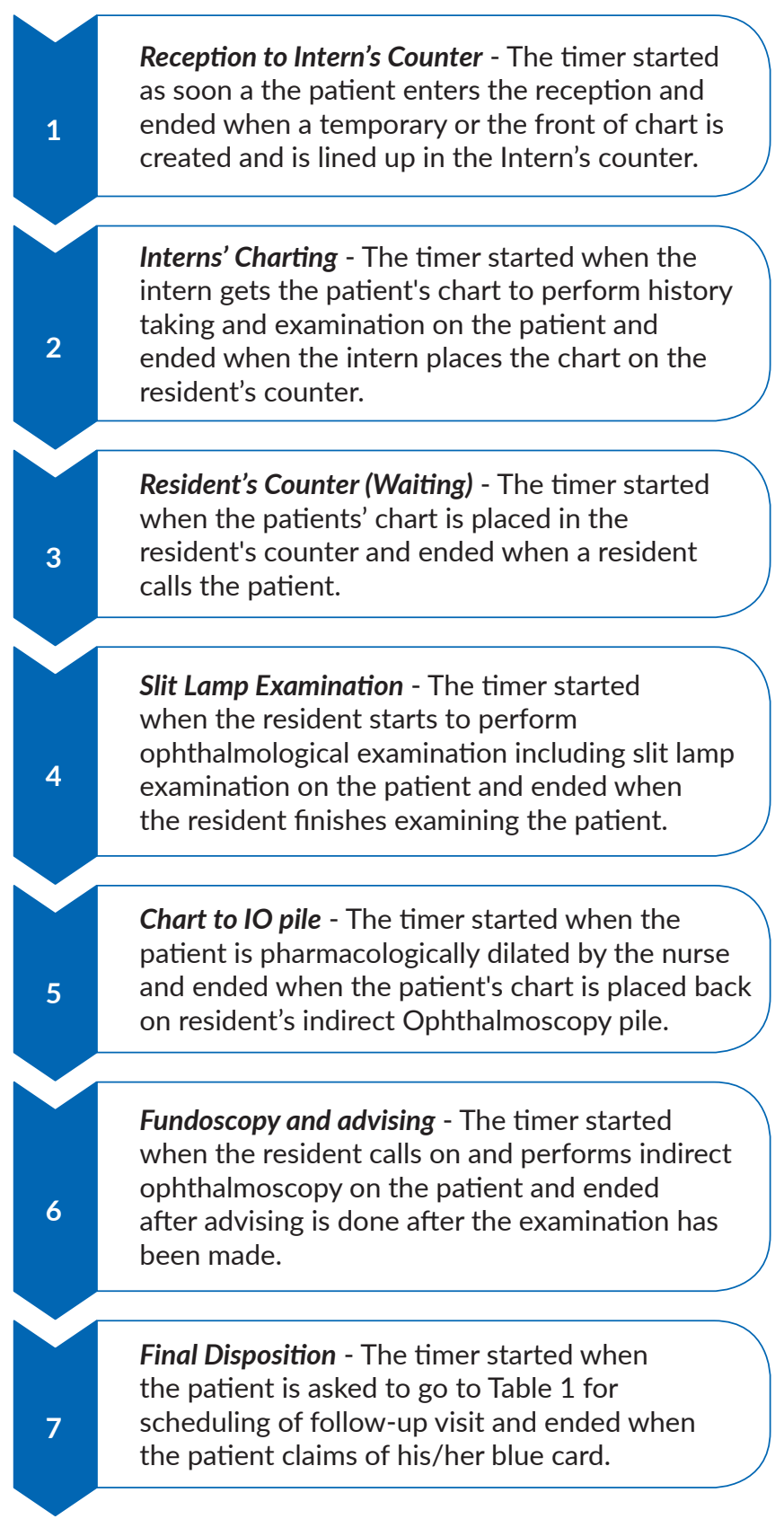

Figure 1. Steps that a new patient undergo in a usual General Clinic Consult.

Consult time of Pre-intervention group - Consult time of Post-intervention group Consult time of Pre-intervention group 


\section{RESULTS}

For the pre-intervention group, 35 new patients were tracked. It took a median of 220 minutes ( 3.7 hours) for the new patients to complete all the steps in a General Clinic consult (Figure 2). These data were presented to the team, and root cause analysis of the possible causes of prolonged consultation visit were done.

During the root cause analysis, many factors were identified as possible causes of prolonged consultation visit. These factors were grouped into records, interns, residents, and patients (Table 1).

Interventions were discussed, planned and instituted. Communication with the Record Section was done to address the problem with the late arrival of front of charts and blue cards at the General Clinic. A proposal of having a separate record section for printing of the front of charts and blue cards in the General Clinic was made, but it was not acceptable to the Record Section personnel. Individual interns' kit including a pinhole and a Jaeger chart was given to each intern rotating in the department, and an Ishihara chart was provided to each block of interns rotating. Awareness of the residents and other staff on ways to make General Clinic consult efficient were increased. The residents were given guidelines on patients who need dilated fundoscopy. Routine dilated fundoscopy of all patients was stopped. Motility patients had cycloplegic refraction in the Subspecialty Clinic. These interventions were instituted for 4 months, after which a post-intervention analysis was done where another 35 new patients were tracked. The summary of the post-intervention group is shown in Figure 3.

Comparisons were made on the median consultation time in each step and the median total time to complete a General Clinic consult for new patients pre-intervention and post-intervention (Table 2). Significant reductions were seen in time spent for steps 1 ( $\mathrm{p}<0.05), 5(\mathrm{p}<0.002)$ and 6 $(\mathrm{p}<0.0001)$. This resulted to a significant reduction in overall consult time $(\mathrm{p}<0.0003)$ of $31 \%$. Time spent in steps 2 and 4 were increased by 2 minutes but were not statistically significant. More than 1 hour was reduced in the consult time after the interventions were implemented.

\section{DISCUSSION}

The highest median reduction in time of consult was seen in step 6 at $15(2.5,40)$ minutes. However, increases in time consult were noted in steps 2 and 4 albeit minimally. Serving patients demands a standard quality of care delivered in an efficient and satisfactory manner. This study showed that the total consultation time of a new patient in the

Table 1. Factors identified contributing to longer consultation of new patients in the GC

\section{Factors}

Records - General Clinic started at 7:00 AM, but patient arrived from main OPD at 7:15-7:20 AM. Temporary charts were usually ready by 7:30 am.

- After the patient was done with all examinations and was for disposition by the assigned nurse at the Table 1 , they still had to wait for the blue card and front of chart to be printed and brought to them from the main OPD, which usually arrived at 10:00-10:30 AM.

\begin{tabular}{cl}
\hline Intern & - Interns complain of insufficient number of pinhole, Ishihara charts and Jaeger charts. \\
\hline Resident & - Although there were 3 residents in the General Clinic, one resident also see Pay-patients and sometimes patients in the \\
& Cataract Clinic. The other two residents have also other assignments in the Subspecialty Clinic simultaneously. \\
& - Residents routinely dilate all patients as part of the examination even without indications. \\
- Residents routinely do cycloplegic refraction of pediatric patients before referring to the Motility service.
\end{tabular}

Table 2. Comparison of Average Time in Minutes in General Clinic Pre-intervention and Post-intervention

\begin{tabular}{|c|c|c|c|c|c|}
\hline \multirow{2}{*}{ Steps } & \multicolumn{2}{|c|}{ Median Consultation time (minutes) } & \multirow{2}{*}{$\begin{array}{l}\text { Median Difference } \\
\text { (minutes) }\end{array}$} & \multirow{2}{*}{$z$-value, $p$-value } & \multirow{2}{*}{$\begin{array}{l}\text { Median Time } \\
\text { reduction (\%) }\end{array}$} \\
\hline & Pre-intervention group & Post-intervention group & & & \\
\hline 1 - Reception to Intern's Counter & $5(3,12.5)$ & $5(0,10)$ & $2(-2,6)$ & $-1.916,0.05^{*}$ & $33(-45,100)$ \\
\hline 2 - Interns' Charting & $30(24,37.5)$ & $30(25,37.5)$ & $-2(-7.5,14)$ & $-0.106,0.92$ & $-6(-40,40)^{* *}$ \\
\hline 3 - Resident's Counter (Waiting) & $25(10,44.50$ & $20(12.5,24)$ & $1(-7.5,32.5)$ & $-1.447,0.15$ & $11(-92,88)^{* *}$ \\
\hline 4 - Slit Lamp Examination & $12(7,21)$ & $15(10,20)$ & $-2(-5,5)$ & $1.02,0.31$ & $-15(-74,27)^{* *}$ \\
\hline 5 - Chart to IO pile & $25(20,30)$ & $17(0,25)$ & $5(-4,25)$ & $-3.054,0.002^{*}$ & $20(-20,100)$ \\
\hline $6-10$ and Advising & $24(13,45)$ & $10(0,15)$ & $15(2.5,40)$ & $-4.568,0.0001^{*}$ & $87.5(11,100)$ \\
\hline 7 - Final Disposition & $22(10,60)$ & $25(5,90)$ & $0(-66,15.5)$ & $0.082,0.93$ & $0(-294,75)$ \\
\hline Total & $220(155.5,273)$ & $136(117.5,182.5)$ & $64(-2,121.5)$ & $-3.607,0.0003^{*}$ & $31(-1,53)$ \\
\hline
\end{tabular}

*statistically significant

${ }^{* *}$ negative median time reduction indicates prolonged time in that step 


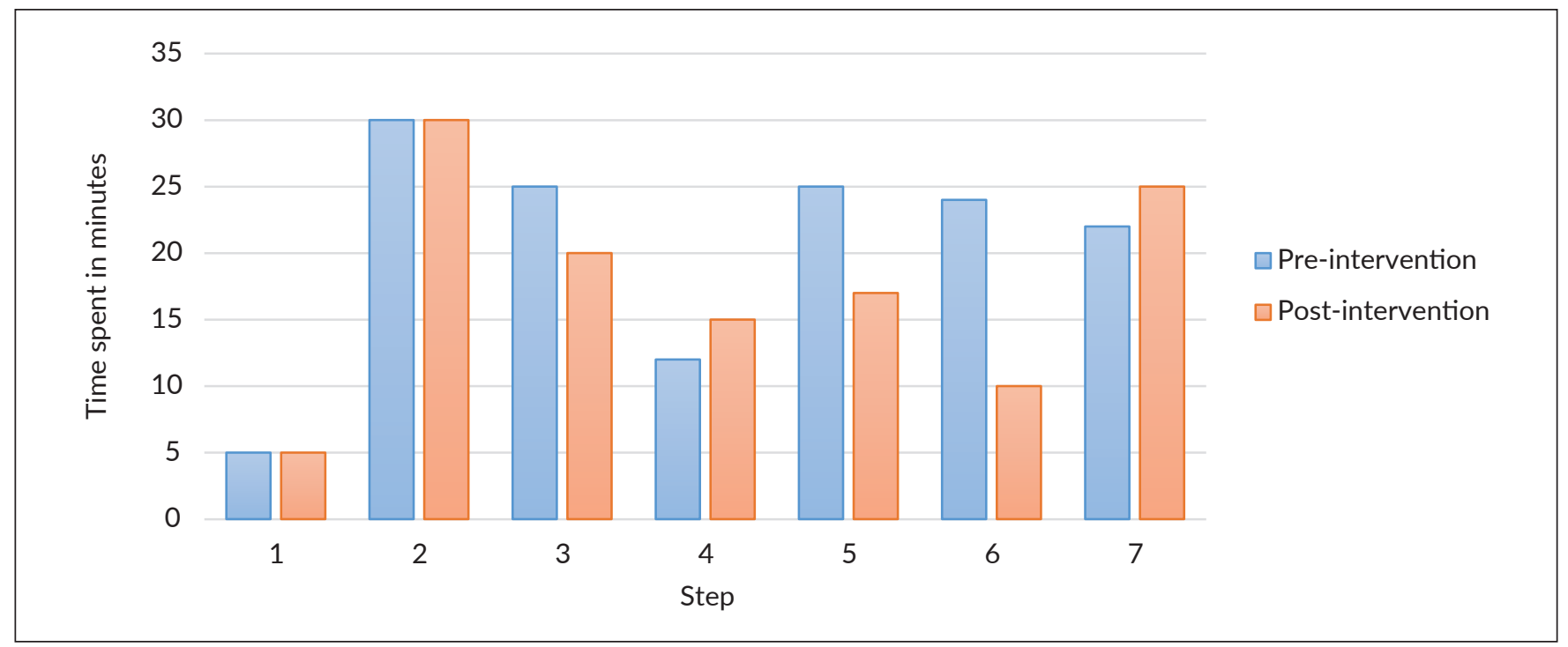

1 - Reception to Intern's Counter; 2 - Interns' Charting; 3 - Resident's Counter (Waiting); 4 - Slit Lamp Examination; 5 - Chart to IO pile; 6 - IO and Advising; 7 - Final Disposition

Figure 2. Comparison of the median time spent in each step of new patients during GC consult in the pre (blue) and postintervention group (orange).

General Clinic decreased with the help of majority of the personnel involved in the eye care delivery system after identifying factors causing the prolonged consultation visit, and instituting interventions to address these identified factors. Improvement in health service delivery was proven to be achievable with the use of time motion study similar to those who have used it before. ${ }^{1-4}$

The ongoing COVID-19 reduced hospital operations including in the General Clinic and shifted consultations to online ones. However, despite the limited operations, patients are starting to consult again. Should the General Clinic patient load return to the pre-pandemic level and become equivalent to the study's pre-intervention status, a different root cause analysis will be needed as causes in delay may have changed already. However, this study demonstrated that a time motion study is effective to address the prolonged consult time for new patients in the General Clinic.

Limitations of this study include being done only in the General Clinic of the department. The authors recommend that the same time-motion study be conducted in subspecialty clinics as well. Institution of intervention with other factors such as Records' and patients' factor may further increase the efficiency of the results.

\section{CONCLUSION}

A time-motion study reduced the total consultation time of new patients in the General Clinic at the Department of Ophthalmology and Visual Sciences of a Philippine Tertiary Hospital by identifying causes and addressing them with the help of the personnel involved in the system.

\section{Statement of Authorship}

All authors contributed in the conceptualization of work; acquisition and analysis of data; drafting and revising; and approved the final version submitted.

\section{Author Disclosure}

All authors declared no conflicts of interest.

\section{Funding Source}

This study has no funding support.

\section{REFERENCES}

1. Sengupta M, Chakrabarti S, Mukhopadhyay I. Waiting Time: The Expectations and Preferences of Patients in a Paediatric OPD. J Health Manag. 2019;21(3):427-442.

2. Harper S, Mousa F. Time and Motion Studies [Internet]. oxford bibliographies. 2013 [cited 28 June 2021]. Available from: https:// www.oxfordbibliographies.com/view/document/obo-9780199846740/ obo-9780199846740-0027.xml

3. Lopetegui M, Yen P, Lai A, Jeffries J, Embi P, Payne P. Time motion studies in healthcare: What are we talking about?. J Biomed Inform. 2014;49:292-299.

4. Deepika S, Parande A, Surwade J, Tapare V, Tambe M, Bhattacharya S. A Time Motion Study in The Immunization Clinic of A Tertiary Care Hospital, BJGMC Pune. Indian J Public Health Res Dev. 2017;8(4):892.

5. Singh R, Talwar Y, Kumar A. A Time and Motion Study of Patients Presenting at the Accident and Emergency Department at Advanced Paediatric Centre of Pgimer, Chandigarh. Health Sci J. 2019;13(1):635. doi: 10.36648/1791-809X.1000635 